\title{
AVALIAÇÃO DA UTILIZAÇÃO DE RESÍDUOS INCORPORADOS AO CLÍNQUER PORTLAND: REVISÃO SISTEMÁTICA
}

Thamires Rodrigues de Oliveira1; Luara Batalha Vieira2; Larissa da Silva Paes Cardoso $^{3}$; Josiane Dantas Viana ${ }^{4}$

${ }^{1}$ Senai - Cimatec; Salvador/BA; thamires.deoliveira@icloud.com

2 Senai - Cimatec; Salvador/BA

${ }^{3}$ Senai - Cimatec; Salvador/BA

${ }^{4}$ Senai - Cimatec; Salvador/BA

Resumo: A Indústria de cimento gera grandes volumes de extração de recursos naturais utilizados como matérias-primas, além da emissão de grandes quantidades de $\mathrm{CO}_{2}$ e outros gases poluentes liberados no processo de queima em altas temperaturas. Sob outra perspectiva, o setor tem grande capacidade de reaproveitamento de resíduos em seu processo produtivo. Sendo assim, o presente trabalho utiliza-se do método de pesquisa sistemática realizado nas plataformas ScienceDirect, Portal de Periódicos Capes e Google Acadêmico, com o objetivo de analisar as contribuições acadêmicas acerca da utilização de resíduos no processo de produção de clínquer, o principal constituinte do cimento. A pesquisa permite a conclusão de que a utilização de resíduos como matéria-prima para fabricação do cimento é possível, economicamente viável e traz diversos benefícios técnicos e sócioambientais.

Palavras-Chave: Resíduo Industrial; Clínquer Portland; Coprocessamento; Pó de mármore; Celulose.

\section{EVALUATION OF THE USE OF PORTLAND CLINQUER WASTE: SYSTEMATIC REVIEW}

\begin{abstract}
The cement industry generates large amounts of extraction of natural resources used as raw materials, in addition to the emission of large amounts of $\mathrm{CO}_{2}$ and other pollutant gases released in the process of burning at high temperatures. From another perspective, the sector has a large capacity to reuse waste in its production process. This, the present work uses the systematic research method carried out on the platforms ScienceDirect, Capes and Google Scholar in order to analyze the literary contributions about the use of waste in the clinker production process, the main constituent of cement. The research allows the conclusion that the use of waste as a raw material for cement manufacturing is possible, ecologically and economically viable.
\end{abstract}

Keywords: Industrial Waste; Portland Clinker; Co-processed; marble powder; Cellulose. 


\section{INTRODUÇÃO}

A construção civil é de grande importância não só para o Brasil como no mundo, tendo influência direta no desenvolvimento econômico. Com o crescimento populacional e o avanço da tecnologia, há um consequente aumento no número de construções, impulsionando a utilização de cimento, um dos produtos mais importantes do setor.

O consumo de cimento no mundo no ano de 2017 foi estimado em 4,1 bilhões de toneladas e se manteve próximo em relação ao ano anterior [1]. Se tratando apenas da produção de concreto mundial, a utilização de cimento está em torno de 1,6 bilhões de toneladas. A China é o país líder na produção e consumo de cimento com $58,8 \%$ total da produção mundial, seguida da India, países da União Europeia, os Estados Unidos e o Brasil [2].

No entanto, o processo produtivo do cimento gera diversos impactos negativos ao meio ambiente. Para a produção do cimento utiliza-se cerca de $3 \%$ da energia primária do mundo [3], com emissão para a atmosfera em torno de 2 bilhões de toneladas de $\mathrm{CO}_{2}$ [4], além do processo possuir baixo rendimento de conversão, pois para produção de 1 tonelada de clínquer é necessária a extração de 1,5 a 1,7 toneladas de matérias-primas [5,6].

O elemento fundamental do cimento é o clínquer. Pode ser definido como um material obtido por meio da sintetização de calcário, cerca de $80 \%$, e de $20 \%$ de argila ou outro material semelhante. Os materiais são moídos e misturados para que sua composição obtenha teores médios de óxidos fundamentais $[7,8]$. Se apresenta em maior quantidade o óxido de cálcio ( $\mathrm{CaO}$ ), que compõem $58 \%$ a $67 \%$ do total da composição, seguido do óxido de silício $\left(\mathrm{SiO}_{2}\right)$, com $16 \%$ a $26 \%$, o óxido de alumínio $\left(\mathrm{Al}_{2} \mathrm{O}_{3}\right), 4 \%$ a $8 \%$, e o óxido de ferro $\left(\mathrm{Fe}_{2} \mathrm{O}_{3}\right)$, com $2 \%$ a $5 \%$, formando a farinha crua [9]. Esta é aquecida em forno rotativo com elevadas temperaturas, em torno de 1450 ${ }^{\circ} \mathrm{C}$. O calor aplicado modifica a estrutura deste material, transformando-o em clínquer, em seguida o material é resfriado bruscamente para elevação de sua dureza, para assim facilitar o processo de moagem [10]. Outras matérias-primas, como por exemplo, filler e escoria de alto-forno são adicionadas e misturadas ao clínquer, permitindo a fabricação dos diferentes tipos de cimento Portland encontrados no mercado [11].

A geração de resíduos é um problema atual e quando destinado de forma inadequada contribue para a degradação do meio ambiente. A Política Nacional de Resíduos Sólidos (PNRS), Lei no 12.305, trata de diretrizes relacionadas ao gerenciamento dos resíduos, e as responsabilidades dos geradores [12].

A busca por alternativas sustentáveis é de extrema importância. Um dos meios para redução do impacto ambiental tanto no processo produtivo do cimento, como para destinação dos resíduos industriais é o coprocessamento. Trata-se de uma técnica em que os resíduos sólidos são inseridos na fabricação de produtos que necessitam de altas temperaturas [13,14].

Uma das grandes beneficiadas do processo de coprocessamento é a indústria de cimento, pois utiliza-se da incorporação de resíduos junto à matéria-prima para 
diminuição da extração dos recursos naturais e/ou como substituição de combustível, visando a diminuição das emissões dos gases poluentes.

O número de fábricas habilitadas para o uso de resíduos no mundo e no Brasil vem aumentando dos anos 2000 até os dias de hoje. Estão espalhadas em 18 estados, 57 plantas integradas que possuem forno rotativo, 36 são licenciados para 0 coprocessamento de resíduos. As operações no ano de 2015, obtiveram 1,07 milhão de toneladas de resíduos para utilização no processo de fabricação do cimento, porém o setor ainda tem um grande potencial e pode chegar a produzir 2,5 milhões de toneladas. Estes dados podem representar uma alternativa para reduzir e minimizar os impactos ambientais, dando destinação adequada aos resíduos gerados nos processos industriais, trazendo assim grandes benefícios socioeconômicos para diversos setores [2].

Diante deste cenário, alguns estudos vêm sendo desenvolvidos no sentido de se conhecer melhor o potencial de aplicação de diferentes resíduos na produção do clínquer Portland, analisando a viabilidade técnica e econômica e os benefícios ambientais promovidos pela destinação adequada do resíduo a um novo tipo de cimento [15-24]. Neste contexto, o objetivo deste trabalho foi avaliar as contribuições das pesquisas desenvolvidas acerca da incorporação de resíduos industriais no processo de fabricação do clínquer, tendo em vista a identificação de algumas vertentes a serem exploradas, como por exemplo, o aprofundamento das formulações dos clínqueres, considerando o aumento do teor de incorporação dos resíduos.

\section{METODOLOGIA}

O estudo foi realizado através de uma revisão sistemática, em que buscou-se analisar aspectos ligados à utilização de resíduos no processo de fabricação do clínquer, principal constituinte do cimento.

As bases de dados utilizadas para realização das buscas para a revisão sistemática foram: ScienceDirect, Scopus, Portal de Periódicos Capes e Google Acadêmico. Os descritores utilizados foram: Industrial Waste; Portland Clinker; Coprocessed; Marble Powder; Cellulose.

Os critérios de inclusão definidos para a realização da busca das publicações foram: pertinência dos conteúdos em relação ao tema abordado neste trabalho; trabalhos publicados a partir de 2015; trabalhos nos idiomas inglês e português e trabalhos disponibilizados na íntegra. Foram excluídos todos os trabalhos publicados fora do período, dos idiomas definidos e os artigos com disponibilidade mediante pagamento.

A realização da busca nos bancos de dados selecionados se deu através do acesso on-line, entre os meses de fevereiro a julho de 2019. Este trabalho de revisão sistemática incluiu 10 estudos e os números de inclusão e exclusão por base de dados estão detalhados na Tabela 1.

A combinação dos descritores se deu por 3 sentidos e a palavra and foi utilizada como conectivo, conforme apresentado na Tabela 1. Para selecionar os artigos 
utilizou-se inicialmente o título do trabalho, sendo inclusos os títulos aderentes ao tema. Logo em seguida, pelo resumo, em que foram excluídos aqueles que não possuíam aderência aos temas abordados nesta revisão sistemática.

Tabela 1. Apresentação da seleção dos artigos segundo Bases de Dados

\begin{tabular}{|c|c|c|c|}
\hline & ScienceDirect & $\begin{array}{c}\text { Google } \\
\text { Acadêmico }\end{array}$ & $\begin{array}{l}\text { Portal de } \\
\text { Periódicos } \\
\text { Capes }\end{array}$ \\
\hline Descritores & $\begin{array}{l}\text { Total de } \\
\text { trabalhos/lncl } \\
\text { uídos }\end{array}$ & $\begin{array}{l}\text { Total de } \\
\text { trabalhos / } \\
\text { Incluídos }\end{array}$ & $\begin{array}{l}\text { Total de } \\
\text { trabalhos } \\
\text { /lncluídos }\end{array}$ \\
\hline $\begin{array}{l}\text { Industrial Waste AND } \\
\text { clinker Portland AND co- } \\
\text { processed }\end{array}$ & $35 / 0$ & $44 / 2$ & $17 / 0$ \\
\hline $\begin{array}{lcc}\text { Industrial } & \text { waste } & \text { AND } \\
\text { clinker } & \text { Portland } & \text { AND } \\
\text { marble slurry } & & \end{array}$ & $15 / 1$ & $57 / 2$ & $19 / 1$ \\
\hline $\begin{array}{lcr}\text { Industrial } & \text { waste } & \text { AND } \\
\text { Cellulose } & \text { AND } & \text { clinker } \\
\text { Portland } & & \end{array}$ & $14 / 1$ & $111 / 2$ & $40 / 1$ \\
\hline
\end{tabular}

Após a realização da pesquisa, foi possível encontrar o total de 342 estudos pertinentes ao tema, foram selecionados 10 trabalhos para compor esta revisão sistemática após leitura dos títulos e resumo.

Dos trabalhos encontrados na base de dados do Google Acadêmico, dois poderiam ter sido incluídos, porém só estavam disponíveis mediante pagamento. Dos 10 estudos inclusos na revisão sistemática, 8 são artigos científicos e 2 são dissertação de mestrado.

Dos trabalhos analisados os 10 tratam de incorporação de diferentes resíduos no processo produtivo do clínquer. Dentre eles, 2 são de resíduos de minério não reagido (MNR), 4 são de resíduos de mármores e, por fim, 4 resíduos da indústria de celulose.

\section{RESULTADOS E DISCUSSÃO}

\subsection{Características dos resíduos industriais}

Os trabalhos analisados revelam que há grande possibilidade de produzir 0 clínquer com resíduos diferentes por sua versatilidade da queima [15-24]. Os resíduos utilizados são dos setores da indústria de celulose, indústria de pigmentos (resíduos de minério não reagido) e indústria de rochas ornamentais. Os resíduos são 
classificados como não perigosos pertencentes à classe II A, estabelecida pela NBR 10.004 [15-24].

Os resíduos provenientes da indústria da celulose revelam que em sua composição química o óxido de cal $(\mathrm{CaO})$ é predominante no resíduo. Outros elementos também estão presentes como: dióxido de silício $\left(\mathrm{SiO}_{2}\right)$ óxido de alumínio $\left(\mathrm{Al}_{2} \mathrm{O}_{3}\right)$ e óxido de ferro $\left(\mathrm{Fe}_{2} \mathrm{O}_{3}\right)$, entretanto os teores variam dependendo da forma de utilização do resíduo gerado podendo ser em lama calcaria, lama biológica e cinzas volantes da queima da biomassa [15-18].

Já a indústria de pigmentos utiliza como matéria-prima o dióxido de titânio $\left(\mathrm{TiO}_{2}\right)$ ou titânia, um produto de origem natural que está presente na composição da ilmenita $\left(\mathrm{FeTiO}_{3}\right)$ e das escórias de titânio. O resíduo gerado desta produção com alto teor de $\mathrm{TiO}_{2}$ é denominado minério não reagido (MNR). O resíduo possui em sua composição elementos mineralizantes, acelerando as reações no processo de sintetização dos materiais $[19,20]$.

E por fim os resíduos provenientes da indústria de rochas ornamentais. As análises químicas dos resíduos revelam que o óxido de cal, $\mathrm{CaO}$ e o oxido de silício, $\mathrm{SiO}_{2}$ encontram-se em maior valor, dependendo do tipo de rocha. $\mathrm{O}$ teor de cada elemento pode variar de acordo com o método de extração dos blocos podendo conter elementos abrasivo. As rochas podem ser de origem sedimentar, metamórficas ou ígnea [21-24].

\subsection{Características do Clínquer contendo resíduos da indústria de celulose}

Dentre os estudos que avaliaram a incorporação de resíduos da indústria de papel e celulose no processo produtivo do clínquer [15-18], foram realizados ensaios de caracterização química, mineralógica, análise térmica e distribuição granulométrica dos clínqueres experimentais e quando comparados ao clínquer convencional não houveram alterações significativas [15].

As pesquisas de Buruberri $[16,17]$ demonstram que a temperatura do forno para o processo produtivo do clínquer foi menor com a utilização do resíduo da celulose incorporado à mistura, ficando em torno de $1390^{\circ} \mathrm{C}$, reduzindo assim o consumo energético na produção do clínquer. Observou-se que a presença do cloreto nesses resíduos não afetou a qualidade do clínquer em relação à durabilidade do cimento, pois os cloretos foram quase totalmente eliminados durante o processo de queima $[16,17]$.

\subsection{Características do Clínquer contendo resíduos da $\mathrm{TiO}_{2}$}

Foi encontrado apenas dois trabalhos de um mesmo autor, uma dissertação e um artigo, que abordaram o uso de resíduo de minério não reagido (MNR) na produção do clínquer. Para verificar a eficácia da produção de cimento com a incorporação do resíduo, a autora Mariani $[19,20]$ testou em laboratório diferentes teores de incorporação do minério não reagido (MNR), sendo eles de 0,5\%, 1,0\% e $2,0 \%$. Constatou-se que a substituição parcial do calcário pelo minério não reagido necessitou de uma temperatura inferior à usual, que é de $1450^{\circ} \mathrm{C}$, chegando-se a uma 
temperatura de queima ideal de $1340^{\circ} \mathrm{C}$, reduzindo assim o consumo de energia no processo de sintetização dos materiais $[19,20]$.

Foi possível identificar que quantidades superiores a $1 \%$ de incorporação apresentaram deficiência na formação da fase líquida que se cristaliza e envolve os cristais de alita, principal responsável pela resistência mecânica do cimento nas primeiras idades. O teor ideal definido foi o de $0,5 \%$ e o fator de saturação de cal (FSC) foi de $98 \%$, pois as principais fases de clínquer convencional, belita $\left(\mathrm{C}_{2} \mathrm{~S}\right)$, alita $\left(\mathrm{C}_{3} \mathrm{~S}\right)$, aluminato $\left(\mathrm{C}_{3} \mathrm{~A}\right)$ e a ferrita $\left(\mathrm{C}_{4} \mathrm{AF}\right)$ foram formadas, tornando-se uma alternativa viável para a sua utilização $[19,20]$.

\subsection{Características do Clínquer contendo resíduos da indústria de rochas ornamentais.}

Quatro estudos analisaram a incorporação do resíduo mármore [21-24]. O autor Ruiz Sanhcez [21] destaca que os resultados obtidos com a utilização do resíduo mostraram que a composição mineralógica é favorável à sua reutilização, pois é baseada na presença de oxido de cálcio $(\mathrm{CaO})$ e a análise físico-química confirmou sua viabilidade como subproduto puro, limpo e ultrafino. Com o uso de 5 teores de incorporação de mármore (0\%,5\%, 10\%, 15\%, 20\% e 25\%). A partir dos ensaios de caracterização química, mineralógica o teor ideal definido pelo autor para incorporação é de $10 \%$.

Já o autor Kirgiz [22], além da utilização do resíduo de pó de mármore em proporções maiores, incorporou aos clínqueres resíduo de pó ultrafino de tijolos em diferentes teores, sendo eles: $79 \%$ e $21 \%$; $77 \%$ e $23 \%$; e $81 \%$ e $19 \%$. As composições químicas apresentadas tiveram resultados satisfatórios em relação ao baixo teor de óxido de magnésio ( $\mathrm{MgO}$ ). No estudo, o teor ideal de utilização dos resíduos foi de $81 \%$ de pó de mármore e $19 \%$ de pó de tijolo, pois possui uma maior presença dos óxidos de cálcio em sua composição e melhor desempenho mecânico quando comparado ao clínquer industrial.

\section{CONCLUSÃO}

Esta revisão sistemática analisou trabalhos relacionados à incorporação de resíduos industriais ao clínquer.

Para produção do clínquer utiliza-se do forno rotativo em elevadas temperaturas em torno de $1450^{\circ} \mathrm{C}$. O emprego de resíduos neste processo tem como vantagem a queima de matéria orgânica e neutralização de gases perigosos. Neste contexto, torna-se possível a incorporação de resíduos junto à matéria-prima convencional sem alteração das propriedades do produto final. O uso do resíduo contribui para menor consumo de matéria-prima e/ou para redução do consumo enérgico, diminuindo a emissão de $\mathrm{CO}_{2}$ e outros gases poluentes.

Os trabalhos pesquisados avaliaram as características químicas e mineralógicas dos resíduos para possibilitar a realização dos estudos de incorporação de diferentes teores de resíduo antes da sintetização dos materiais. Cada resíduo possui a sua particularidade, teor e diferentes meios de utilização. O resíduo da celulose e o da indústria de pigmentos, além de reduzirem a incorporação da matériaprima convencional, também possibilitaram um menor consumo enérgico, baixando a 
temperatura de queima de $1450^{\circ} \mathrm{C}$ para $1390^{\circ} \mathrm{C}$ e $1340^{\circ} \mathrm{C}$ respectivamente. Já o resíduo de rochas ornamentais possibilitou a substituição de até $81 \%$ da matériaprima.

Embora os presentes trabalhos contribuam para confirmação da utilização dos resíduos para a incorporação ao clínquer, nota-se que são necessárias mais pesquisas sobre o aperfeiçoamento das formulações das misturas entre o resíduo e a matéria-prima convencional, verificação de produção em escala industrial e análise do emprego dos cimentos produzidos em concretos e argamassas para avaliação da durabilidade desses materiais, possibilitando assim uma análise mais consistente do cimento produzido com incorporação de resíduos.

\section{REFERÊNCIAS}

1 U.S. Geological Survey (USGS). Mineral commodity summaries 2018: U.S.

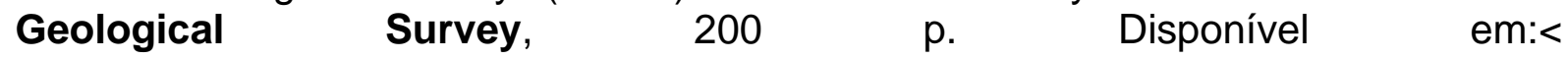
https://minerals.usgs.gov/minerals/pubs/mcs/2018/mcs2018.pdf> Acesso em: 03 de maio de 2018.

2 SNIC. Dados do setor. 2016. Disponível em: <http://snic.org.br/numeros-dosetor.php>. Acesso em: 23 maio 2019.

3 JUENGER, M.c.g. et al. Advances in alternative cementitious binders. Cement And Concrete Research, [s.I.], v. 41, n. 12, p.1232-1243, dez. 2011. Elsevier BV. http://dx.doi.org/10.1016/j.cemconres.2010.11.012.

4 SHI, C.; JIMÉNEZ, A. F.; PALOMO, A. New cements for the 21st century: The pursuit of an alternative to Portland cement. Cement And Concrete Research, [s.I.], v. 41, n. 7, p.750-763, jul. 2011. Elsevier BV. http://dx.doi.org/10.1016/j.cemconres.2011.03.016.

5 YEN, C.; TSENG, D.; LIN, T. Characterization of eco-cement paste produced from waste sludges. Chemosphere, [s.I.], v. 84, n. 2, p.220-226, jun. 2011. Elsevier BV. http://dx.doi.org/10.1016/j.chemosphere.2011.04.050.

6 ZHANG, T. et al. Effectiveness of novel and traditional methods to incorporate industrial wastes in cementitious materials-An overview. Resources, Conservation And Recycling, [s.l.], v. 74, p.134-143, maio 2013. Elsevier BV. http://dx.doi.org/10.1016/j.resconrec.2013.03.003.

7 Taylor W. H. F. Cement chemistry. Londres: Thomas Telford, 1997.

8 PANI, A. K.; MOHANTA, H. K. Online monitoring of cement clinker quality using multivariate statistics and Takagi-Sugeno fuzzy-inference technique. Control Engineering Practice, [s.I.], v. 57, p.1-17, dez. 2016. Elsevier BV. http://dx.doi.org/10.1016/j.conengprac.2016.08.011.

9 DUDA, W. H. Cement data book: international process engineering in the cement industry. 3. ed. Berlin: Wiesbaden, 1985.

10 NEVILLE, A.M. BROOKS, J.J. Tecnologia do Concreto. 2ª Ed., Bookman, 2013. 
11 ASSOCIAÇÃO BRASILEIRA DE CIMENTO PORTLAND. Guia básico de utilização do cimento Portland. Boletim Técnico. São Paulo: ABCP, 2002.

12 BRASIL. Departamento Nacional de Produção Mineral. Sumário mineral. Coordenadores: Thiers Muniz Lima e Carlos Augusto Ramos Neves. Brasília: DNPM, 2014

${ }^{13}$ MARINGOLO, V. Clínquer Coprocessado: Produto de Tecnologia Integrada para Sustentabilidade e Competitividade da Indústria de Cimento. 2001. $174 \mathrm{f}$. Tese (Doutorado) - Curso de Pós-graduação em Mineralogia e Petrologia, Universidade de SÃo Paulo, São Paulo, 2001.

${ }^{14}$ BAIDYA, R.; GHOSH, S. K. P, Ulhas V.. Co-processing of Industrial Waste in Cement Kiln - A Robust System for Material and Energy Recovery. Procedia Environmental Sciences, [s.I.], v. 31, p.309-317, 2016. Elsevier BV. http://dx.doi.org/10.1016/j.proenv.2016.02.041.

${ }^{15}$ CASTRO, F. et al. Utilisation of pulp and paper industry wastes as raw materials in cement clinker production. Int. J. Materials Engineering Innovation, Portugal, v. 1, n. 1, p.74-90, nov. 2009.

${ }^{16}$ BURUBERRI, L. H.; SEABRA, M.p.; LABRINCHA, J.a.. Preparation of clinker from paper pulp industry wastes. Journal Of Hazardous Materials. Portugal, p. 252260. dez. 2015.

${ }^{17}$ BURUBERRI, L. H. Uso de lamas e cinzas da indústria da celulose em clínquer. 2014. Dissertação de Mestrado. Universidade de Aveiro

18 SIMÃO, L. Desenvolvimento e aplicação de ecoclínquer obtido a partir dos resíduos de setor de papel e celulose. 112 f. Dissertação (Mestrado) - Curso de Ciência e Engenharia de Materiais, Universidade do Extremo Sul do Catarinense, Criciúma, 2016.

${ }^{19}$ MARIANI, B. B. et al. Efeito da incorporação de resíduo de TiO2 (MNR) na formação das fases mineralógicas de clínquer Portland. Ambiente Construído, [s.I.], v. 19, n. 1, p.57-71, mar. 2019.FapUNIFESP (SciELO). http://dx.doi.org/10.1590/s167886212019000100293.

${ }^{20}$ MARIANI, B. B. Produção de clínquer Portland com baixa emissão de $\mathrm{CO} 2$ a partir da incorporação de minério não reagido (MNR) advindo da produção de TiO2. 2018. 160 f. Dissertação (Mestrado) - Curso de Programa de PÓs-graduaÇÃo em Engenharia Civil, Universidade Federal da Bahia Escola PolitÉcnica, Bahia, 2018.

${ }^{21}$ RUIZ S, A.; SÁNCHEZ-POLO, M.; ROZALEN, M.. Waste marble dust: An interesting residue to produce cement. Construction And Building Materials, [s.l.], v. 224, p.99108, nov. 2019. Elsevier BV. http://dx.doi.org/10.1016/j.conbuildmat.2019.07.031.

${ }^{22} \mathrm{KIRGIZ}, \mathrm{M}$. S. Use of ultrafine marble and brick particles as raw materials in cement manufacturing. Materials And Structures, [s.I.], v. 48, n. 9, p.2929-2941, 1 jul. 2014. Springer Science and Business Media LLC. http://dx.doi.org/10.1617/s11527-0140368-6.

${ }^{23}$ AYDIN, E; AREL, H. Ş. High-volume marble substitution in cement-paste: Towards a better sustainability.2019. Disponível em: 
$<$ https://www.sciencedirect.com/science/article/pii/S0959652619326617>. Acesso em: 17 jul. 2019

24 TOPÇU, I. B.; BAYLAVLI, $\mathrm{H}$. The use of concrete wastes as a limestone replacement in limestone-blended cement production. 2019. Disponível em: $<$ https://journalskuwait.org/kjs/index.php/KJS/article/view/4635/323>. Acesso em: 14 jun. 2019 